of sixty-two residues. Its amino-acid sequence has been determined and its toxicity is dependent on the integrity of four disulphide cross-links. Neurotoxins from sea snakes and the Cape cobra are apparently related in structure to cobrotoxin.

This volume should be a useful addition to the shelves of those interested in cellular and molecular developments in this specific area of research.

The chief section of Pharmacology and Toxicology of Naturally Occurring Toxins (which is one in an extensive series) is devoted to toxins from marine organisms, from "red blooms" to sting rays, and the therapeutic problems arising from these are extensively discussed. The venoms of insects and arachnida, from honey bees to scorpions, constitute an astonishing variety of toxic agents and symptoms, but these are reviewed in less detail. The section on mycotoxins is also short, but such a deficiency is more than made up elsewhere (Microbial Toxins, volume VI, v. supra). Although the three groups of toxins are described by different authors with differing styles the book is very easy to read and is supplemented by extensive lists of references. R. J. C. HARrIS

\section{The Dioscoreaceae}

Anatomy of the Monocotyledons. VI. Dioscoreales. By E. S. Ayensu. Edited by C. R. Metcalfe. Pp. xii $+182+16$ plates. (Clarendon: Oxford ; Oxford University: London, March 1972.) £6.

OF the volumes in this important series on the anatomy of the monocotyledons, two have been written by Dr Metcalfe, and all the others have been edited and inspired by him. The volume under review, by Dr Ayensu of the Smithsonian Institution, is a worthy member of the series; it deals with an order with many climbing plants, which is distinctive in structure and which is also of economic importance, containing as it does the cultivated yam. Recently, too, a precursor of cortisone and of steroidal hormones has been shown to be present in some species of Dioscorea.

In Part $\mathbf{I}$, a general account is given of the morphology and anatomy of the order. The account is based on a representative sample of 130 species, belonging to nine genera and four families. There is much to interest the general botanist in this part of the book. It is easy to forget for how short a time and by how few people the families of flowering plants have been studied; and the author reminds us that we still have a lot to learn. For example, some species of the order have two cotyledons, and there is controversy about their nature and morphology. Again, the structure of the xylem- and phloem- glomeruli in the nodes of the Dioscoreaceae seems to be unique in the monocotyledons; these glomeruli are well described by the author, who suggests that their functional significance should be investigated. At the end of Part I there is a valuable short chapter on general taxonomic conclusions based on anatomical evidence, but taking into account other evidence as well; and it is shown that anatomical characters have significance at family, genus and section levels.

In Part II, the families, genera and, in Dioscorea, the subgenera and sections are systematically described. The many line-drawings in the text-figures are carefully executed, but in many cases, where a whole vascular bundle is illustrated at a magnification of $\times 185$, the cells of the ground tissue are so tiny that they can hardly be clearly shown; and one wonders if diagrammatic representation would not have been better. Some of the sections in the photographs, too, are darkly stained and present a rather muddy appearance.

The book is well printed, though a line of text seems to be missing at the bottom of page 2. There is a good index.

D. H. Valentine

\section{Coping with Heat}

The Stress of Hot Environments. By D. McK. Kerslake. Pp. $x+316$. (Cambridge University: London, May 1972.) £6; $\$ 19.50$.

A TEXT taking a long cool look at the physical and physiological basis of thermal stress indices has been long overdue and Dr Kerslake is undoubtedly one of the most suited for such a task. Taking the basic physical data concerning heat exchange between the body and its environment, the physiological data relating to heat transfer within the body and the production of sweat, Kerslake builds up a detailed theory of heat stress against which to compare a number of well known indices. The author does not spare the reader, there are no wasted words, the mathematics is copious and the continual development of ideas requires the reader's full attention. The book is written in a clear style developing concepts in easily grasped steps which should enable all to follow. The twentytwo pages of references, an index and the use of SI units throughout make it a useful reference book.

The first four chapters cover heat transfer and the heat balance of the sweating skin. The development of mean heat exchange coefficients for convection and evaporation is undertaken with detailed reference to the literature and their treatment, and that of radiant heat transfer, should motivate many physiologists to examine their ideas in this area. In radiant heat transfer calculations it seems to be common practice to use the first instead of the fourth power relation, a procedure which is difficult to justify on the grounds of simplifying the calculations as the fourth power can be handled with logarithms or the calculations quickly done using a radiation slide rule. Kerslake examines the simplification and puts its use into perspective, namely to facilitate the joint treatment of radiation and convective heat exchange and the derivation of an operative temperature. Correctly done, making the radiant heat transfer equation linear is by no means a simplification.

The factors which influence and modify the heat exchanges of the body and have to be considered in the development of thermal indices, clothing, respiratory loss and the physiological responses are then covered. In considering the regulation of body temperature, Marius Nielsen's work of 1938 is quoted as proposing that the setting of the body's thermostat, that is, control mechanism, depends on the rate of work. This over-simple thermostat resetting approach to temperature regulation serves only to confuse and it is time it was buried.

The development of temperature regulation follows the normal pattern apart from the idea that heat flow itself may be monitored. This concept is brought in to explain similar sweat rates observed in stepwise increments of radiant heat load and sinusoidal radiant heat loads of progressively larger amplitude to match the values used in the steps. In view of the differences in the rates of change and absolute values of skin temperature in the two situations, this is insufficient reason for proposing the regulation of heat loss.

Having covered the basic groundwork the author sets about integrating the data and reducing the various components which contribute to thermal stress to lines of equal sweat rate (isohids) on a psychrometric chart. The isohids are then used to evaluate some of the commonly used indices. The final chapter covers storage limited heat exposure.

The virtue of the book is the detailed physical analysis of the heat exchanges between man and his environment; its weakness is that the manuscript was finalized at the end of 1970 just about the time P. O. Fanger published his book on thermal comfort and before the 1971 International Symposium on Environmental Physiology. Both events date the book and it is a pity Kerslake did not devote a few pages to a consideration of Fanger's comfort equation. Nevertheless, an excellent book and one to be recommended to all environmental physiologists.

G. W. CROCKFORD 